\title{
Effect of Attention Training in Mild Cognitive Impairment Patients with Subcortical Vascular Changes: The RehAtt Study
}

\author{
Leonardo Pantoni ${ }^{\mathrm{a}, *}$, Anna Poggesi ${ }^{\mathrm{a}}$, Stefano Diciotti ${ }^{\mathrm{b}}$, Raffaella Valenti ${ }^{\mathrm{a}}$, Stefano Orsolini ${ }^{\mathrm{b}}$, \\ Eleonora Della Rocca ${ }^{\mathrm{c}}$, Domenico Inzitari ${ }^{\mathrm{a}, \mathrm{d}}$, Mario Mascalchi ${ }^{\mathrm{e}, \mathrm{f}}$ and Emilia Salvadori ${ }^{\mathrm{a}}$ \\ ${ }^{a}$ NEUROFARBA Department, Neuroscience Section, University of Florence, Florence, Italy \\ ${ }^{\mathrm{b}}$ Department of Electrical, Electronic, and Information Engineering 'Guglielmo Marconi', \\ University of Bologna, Cesena, Italy \\ ${ }^{\mathrm{c}}$ Stroke Unit, Azienda Ospedaliero Universitaria Careggi, Florence, Italy \\ ${ }^{\mathrm{d}}$ Institute of Neuroscience, Italian National Research Council, Florence, Italy \\ ' 'Mario Serio' Department of Experimental and Clinical Biomedical Sciences, University of Florence, \\ Florence, Italy \\ ${ }^{\mathrm{f}}$ Quantitative and Functional Neuroradiology Research Program at Meyer Children Hospital and Careggi \\ General Hospital, Florence, Italy
}

Handling Associate Editor: Daniela Galimberti

Accepted 12 July 2017

\begin{abstract}
.
Background and Objective: Mild cognitive impairment (MCI) patients with small vessel disease (SVD) are at high dementia risk. We tested the effects of cognitive rehabilitation in these patients using the Attention Process Training-II (APT-II) program in a single-blinded, randomized clinical trial.

Methods: Patients were randomized to APT-II or standard care and evaluated at baseline, 6, and 12 months with functional, quality of life, cognitive tests, and resting state functional MRI (rsfMRI).

Results: Forty-six patients were enrolled and 43 (mean \pm SD age $75.1 \pm 6.8$ ) completed the study. No change was seen in functionality and quality of life between treated and non-treated patients. However, the Rey Auditory-Verbal Learning Test immediate recall showed a significant improvement in treated compared to non-treated group (change score 6 versus 12 months: $1.8 \pm 4.9$ and $-1.4 \pm 3.8, p=0.021$; baseline versus 12 months: $3.8 \pm 6.1$ and $0.2 \pm 4.4, p=0.032$ ). A higher proportion of treated patients had stable/better evaluation compared to non-treated group on Visual search test (6 versus 12 months: 95\% versus 71\%, $p=0.038$ ) and Rey-Osterrieth Complex Figure copy (6 versus 12 months: 95\% versus 67\%, $p=0.027)$. RsfMRI, performed in a subsample, showed that the difference between follow-up and baseline in synchronization of activity in cerebellar areas was significantly greater in treated than in non-treated patients.

Conclusion: We were unable to show a significant effect in quality of life or functional status in treated patients with MCI and SVD. However, APT-II produces some beneficial effects in focused attention and working memory and seems to increase activity in brain circuits involved in cognitive processes.
\end{abstract}

Keywords: Cerebrovascular disorders, clinical trial, cognitive dysfunction, functional magnetic resonance imaging, rehabilitation

\footnotetext{
${ }^{*}$ Correspondence to: Leonardo Pantoni, MD, PhD, NEUROFA RBA Department, Neuroscience section, University of Florence,
} 


\section{INTRODUCTION}

Cerebral small vessel disease (SVD) is a leading cause of cognitive impairment [1-3]. Patients with SVD and mild cognitive impairment (MCI) are at high risk of transition to dementia [4]. Therapeutic approaches to reduce this risk are scanty and no drug is approved to obstacle this transition. Cognitive rehabilitation or training may therefore be options in this regard.

The Attention Process Training-II (APT-II) program consists of a group of hierarchically organized tasks aimed at exercising different components of attention (focused, sustained, selective, alternating, and divided) [5]. Because the APT-II contains specific exercises to facilitate generalization to daily life, the skills learned during the training are expected to generalize to daily activities and thus ameliorate patient's overall quality of life.

In this study, we aimed at investigating: 1) the effectiveness of the APT-II program on the rehabilitation of attention in patients with MCI with SVD; 2) the effect of the possible cognitive improvement in real life, in terms of functionality in daily activities and quality of life; 3 ) the possible impact of the attention training on brain activity evaluated with resting state functional MRI (rsfMRI).

\section{MATERIALS AND METHODS}

The RehAtt (Rehabilitation of Attention) study is a 3-year prospective, single-blinded, randomized clinical trial. Its rationale and methodology were extensively reported elsewhere [6]. Methodological details concerning sample size justification, method of randomization, and interventions are detailed in the Supplementary Material.

The study was conducted in accordance with the Helsinki Declaration and was approved by the local ethics committee; each patient gave a written informed consent. The study is registered in the ClinicalTrials.gov registry (identifier number: NCT0203 3850).

Included patients were diagnosed as affected by MCI, with an attentional deficit, and SVD according to the following criteria: 1) MCI according to Winblad et al.'s criteria [7] and operationalized according to Salvadori et al. [8]; 2) evidence of impairment across attention neuropsychological tests (i.e., at least one score borderline among attention/executive functions tests); 3 ) evidence on MRI of moderate to severe age-related white matter hyperintensities (WMH) according to a modified Fazekas scale [9]. The degree of WMH severity was rated on Fluid Attenuated Inversion Recovery (FLAIR) sequences, taking into account only deep and subcortical white matter lesions. The modified Fazekas scale is a visual scale based on a categorization into 3 severity classes: grade $1($ mild $\mathrm{WMH})=$ single lesions below $10 \mathrm{~mm}$, areas of 'grouped' lesions smaller than $20 \mathrm{~mm}$ in any diameter; grade $2($ moderate $\mathrm{WMH})=$ single lesions between 10 and $20 \mathrm{~mm}$, areas of 'grouped' lesions more than $20 \mathrm{~mm}$ in any diameter, no more than 'connecting bridges' between individual lesions; grade 3 $($ severe $\mathrm{WMH})=$ single lesions or confluent areas of hyperintensity $20 \mathrm{~mm}$ or more in any diameter. Exclusion criteria were: 1) inability or refusal to undergo brain MRI; 2) inability to give an informed consent; 3) age $<18$ y.

At baseline, according to the study protocol [6], each patient underwent an extensive clinical, functional, and neuropsychological assessment and an MRI examination. The neuropsychological evaluation was carried out using the VMCI-Tuscany neuropsychological battery [10].

After baseline assessment, participants were randomly assigned to the intervention group (attention training) or the control group (standard care), according to a stratified randomization (see Supplementary Material) [6].

Participants in the intervention group were scheduled to receive a total of $40 \mathrm{~h}$ (2-h weekly sessions for 20 weeks) of individual cognitive training by means of the APT-II program. All cognitive training sessions were administered by a clinical neuropsychologist. Participants in the control group did not receive cognitive interventions, were instructed to have a usual lifestyle, and were provided of medication and clinic consultations as usually needed (see Supplementary Material for details on interventions).

The study protocol comprises follow-up visits at 6 and 12 months. At the follow-up visits, clinical, functional, and neuropsychological assessment were the same of the baseline protocol. The MRI assessment was repeated at 1-year follow-up. The neurologist who collected clinical, functional and neuropsychological data was blind to the intervention conditions. Participants could not be kept blind to the intervention conditions, but they were asked not to reveal their group membership during follow-up assessments, and the neurologist remained blind to training allocation for the entire study duration. 
MRI examination protocol, rsfMRI data preprocessing, regional homogeneity (ReHo) maps computation, and statistical analysis of rsfMRI data are detailed in the Supplementary Material.

\section{Outcome measures}

\section{Primary outcomes}

The primary outcomes were the improvement of functionality in activities of daily living and quality of life in treated compared to non-treated patients. Since this was a pilot study, we decided to assess each outcome with more than one scale.

Functional status was measured by means of three scales administered to the caregiver:

- Activities of Daily Living scale (ADL); score range 0-6 (higher scores represent less disability) [11].

- Instrumental Activities of Daily Living scale (IADL); score range 0-8 (higher scores represent more disability) [12].

- Disability Assessment in Dementia scale; total score in percentage (higher scores represent less disability) [13].

Quality of life was measured by means of four questionnaires on perceived health and/or cognitive status administered to the patient:

- Short Form Health Survey (SF-36); 2 summary scores (Physical and Mental Component Summary) on a 0-100 scale (higher scores represent less disability) [14].

- EuroQol; single summary index and visual analogue 0-100 scale (higher scores represent better health status) [15].

- Attention Questionnaire; score range 0-36 (higher scores represent more presence of attention problems) [5].

- Geriatric Depression Scale; score range 0-15 (higher scores represent more presence of depressive symptoms) [16].

All the functional and quality of life scales are detailed in the Supplementary Material.

\section{Secondary outcomes}

Secondary outcomes included:

1) Improvement in cognitive performance in any of 14 scores deriving from the 11 neuropsychological tests included in the VMCI-Tuscany neuropsychological battery [10], detailed in the Supplementary Material. Cognitive performance of each patient was classified as 'normal', 'borderline' (an age- and educationadjusted score between the outer and inner confidence limits for the 5 th centile of the normal population), or 'abnormal' (an age- and education-adjusted score below the outer confidence limit for the 5th centile of the normal population).

2) Reduction of the risk of transition to dementia. Data collected during the 1-year follow-up visit were used to evaluate the occurrence of a transition from MCI to dementia according to DSM-V criteria [17].

3) Improvement in long-term brain activity as measured by means of ReHo of rsfMRI data, as a result of a training-induced cognitive plasticity, evaluated six months after the end of the cognitive rehabilitation period (see Supplementary Material for further details).

\section{Statistical analysis}

Descriptive analyses were used to illustrate the baseline total sample characteristics. To verify if the attention training and standard care groups differed in baseline characteristics other than those included in the stratified randomization, independent sample $t$ tests and chi square tests were used to compare demographics, vascular risk factors, and global cognitive and functional status.

Primary outcomes and cognitive secondary outcomes were analyzed according to two different approaches:

- Changes in scores $(\Delta)$ approach. Delta scores $(\Delta s)$ were calculated by computing the difference between the scores obtained in two evaluations (baseline versus 6 months; 6 versus 12 months; baseline versus 12 months) for each patient. All $\Delta \mathrm{s}$ were calculated in order that a positive score indicates an improvement, while a negative score indicates a worsening. Delta scores were analyzed using independent sample $t$ tests with treatment (attention training versus standard care) as the only independent variable.

- Clinically significance approach. The availability of $t$ scores for the SF-36 Physical and Mental Component Summary scores allowed us to classify each patient evaluation as "normal well-being' ( $t$ score $>40$ ) or 'reduced wellbeing' $(t$ score $\leq 40)$ at each visit. As previously 
detailed in the secondary outcomes section, the availability of national norms for the cognitive variables allowed us to classify each patient's performance as 'normal', 'borderline', or 'abnormal' at each visit. Variations in performance categories over time (baseline versus 6 month; 6 versus 12 month; baseline versus 12 month) were evaluated for each patient and dichotomized as: 'stable or better evaluation' or 'worse evaluation'. Variations in performance categories were analyzed using chi square tests.

For the analyses on primary and cognitive secondary outcomes, effect sizes were estimated by means of the unbiased Hedges' $g$ (a variation of Cohen's $d$ corrected for biases due to small sample sizes) for the independent sample $t$ tests, and by means of the Phi coefficient $(\varphi)$ for the chi square tests.

Chi square test for a $2 \times 2$ contingency table was used to compare patients who became demented at 1-year follow-up visit with those who did not, in the two treatment groups.

Statistical analysis of rsfMRI data was carried out by feeding Z-transformed ReHo data into voxelwise inter-subject statistics using permutation-based nonparametric inference (randomize, also part of FSL) within the general linear model (GLM) framework. Specifically, to evaluate the possible effect of the treatment, we performed a voxel-wise betweengroup comparison of the difference between the ReHo at follow-up and baseline between treated and non-treated patients (see Supplementary Material for further details).

\section{RESULTS}

The enrolment started on October 1, 2013 and was completed on April 30, 2015. To reach the foreseen number of 40 patients, we evaluated 175 potentially eligible patients. Of these, 129 (74\%) were excluded. As shown in Fig. 1, main reasons for non-enrolment

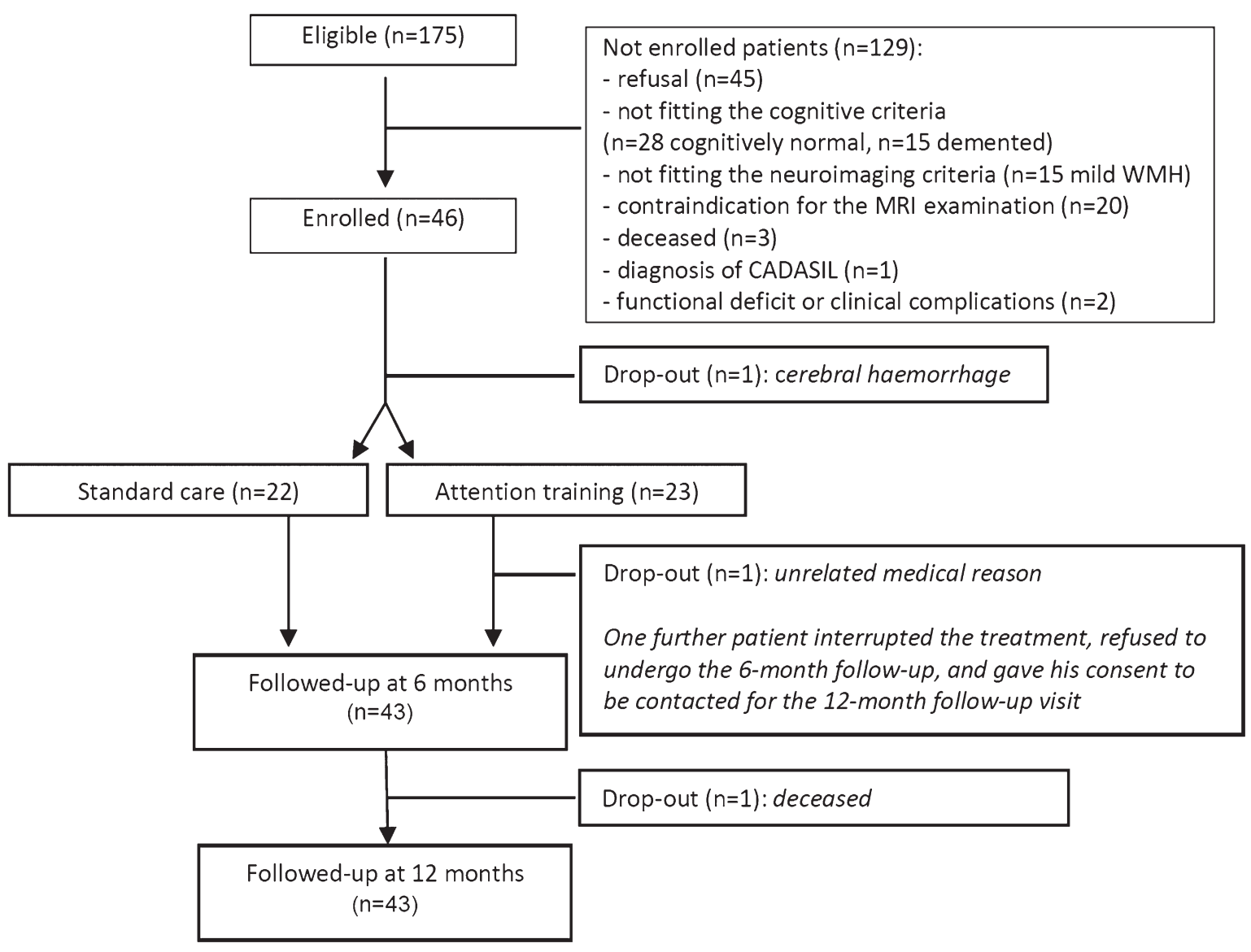

Fig. 1. Flow diagram showing patients' attrition from the screening phase to follow-up assessments. 
Table 1

Total sample characteristics and comparisons between attention training and standard care groups at baseline

\begin{tabular}{|c|c|c|c|c|c|}
\hline & Score range & $\frac{\text { Total sample }}{n=43}$ & $\frac{\text { Attention training }}{n=21}$ & $\frac{\text { Standard care }}{n=22}$ & $p$ \\
\hline Age, y $($ mean \pm SD $)$ & - & $75.1 \pm 6.8$ & $74.2 \pm 6$ & $75.9 \pm 7.6$ & $0.417^{*}$ \\
\hline Education, y & - & $8.2 \pm 4.3$ & $9 \pm 5.3$ & $7.4 \pm 3$ & $0.232 *$ \\
\hline Sex (\% males) & - & $65 \%(n=28)$ & $62 \%(n=13)$ & $68 \%(n=15)$ & $0.666^{\#}$ \\
\hline Hypertension & - & $88 \%(n=38)$ & $86 \%(n=18)$ & $91 \%(n=20)$ & $0.595^{\#}$ \\
\hline Hypercholesterolemia & - & $72 \%(n=31)$ & $76 \%(n=16)$ & $68 \%(n=15)$ & $0.558^{\#}$ \\
\hline Diabetes & - & $16 \%(n=7)$ & $14 \%(n=3)$ & $18 \%(n=4)$ & $0.729^{\#}$ \\
\hline Smoking habits & - & $42 \%(n=18)$ & $52 \%(n=11)$ & $32 \%(n=7)$ & $0.172^{\#}$ \\
\hline History of stroke & - & $40 \%(n=17)$ & $33 \%(n=7)$ & $45 \%(n=10)$ & $0.416^{\#}$ \\
\hline Alcohol consumption & - & $44 \%(n=19)$ & $43 \%(n=9)$ & $45 \%(n=10)$ & $0.864^{\#}$ \\
\hline Mini Mental State Examination & $0-30$ & $26.4 \pm 3$ & $27.1 \pm 2.6$ & $25.7 \pm 3.2$ & $0.132 *$ \\
\hline Montreal Cognitive Assessment & $0-30$ & $19.2 \pm 4.6$ & $19.9 \pm 4.8$ & $18.6 \pm 4.4$ & $0.360^{*}$ \\
\hline Activities of Daily Living (preserved items) & $0-6$ & $5.9 \pm 0.4$ & $5.9 \pm 0.3$ & $5.9 \pm 0.4$ & $0.970^{*}$ \\
\hline Instrumental Activities of Daily Living (impaired items) & $0-8$ & $2.1 \pm 2.2$ & $1.9 \pm 2.1$ & $2.2 \pm 2.4$ & $0.639^{*}$ \\
\hline Disability Assessment in Dementia & $0-100$ & $88 \pm 15.5$ & $91.9 \pm 11.9$ & $84.2 \pm 17.8$ & $0.101^{*}$ \\
\hline Attention Questionnaire & $0-36$ & $15.5 \pm 9.9$ & $15.1 \pm 11.5$ & $15.9 \pm 8.5$ & $0.793^{*}$ \\
\hline EuroQol (summary index) & $-1 /+1$ & $0.7 \pm 0.3$ & $0.7 \pm 0.3$ & $0.7 \pm 0.3$ & $0.491^{*}$ \\
\hline EuroQol (visual scale) & $0-100$ & $65.2 \pm 17.8$ & $63.1 \pm 18.3$ & $67.3 \pm 17.5$ & $0.449^{*}$ \\
\hline Geriatric Depression Scale & $0-15$ & $4.8 \pm 3.8$ & $4.6 \pm 4$ & $4.9 \pm 3.8$ & $0.748^{*}$ \\
\hline
\end{tabular}

*Independent sample $t$ tests. ${ }^{\#}$ Chi square tests.

were: refusal $(n=45)$, not fitting the cognitive criteria ( $n=28$ normal, $n=15$ demented), contraindications for the MRI examination $(n=20)$, not fitting the MRI criteria $(n=15)$. Forty-six patients $(26 \%)$ were finally enrolled and thoroughly assessed according to the study protocol.

Out of the 46 enrolled patients, 1 dropped-out before randomization because of cerebral hemorrhage, and 2 dropped-out after the allocation to the treatment group: one after a total of $4 \mathrm{~h}$ for a gastrointestinal perforation that required emergency surgery and a long hospitalization, while another interrupted the treatment after $30 \mathrm{~h}$ and refused to undergo the 6-month follow-up; he consented to be contacted for the 12-month follow-up visit but deceased before due to a metastatic liver cancer (Fig. 1).

The final RehAtt cohort includes 43 patients affected by MCI with SVD; 22 were randomized to the standard care group and 21 to the attention training group. All these patients completed the protocol, were reassessed at 6 and 12 months, and repeated the MRI exams (Fig. 1).

As shown in Table 1, 65\% $(n=28)$ of the total basal sample were males, and the mean $( \pm \mathrm{SD})$ age and years of education were $75.1 \pm 6.8$ and $8.2 \pm 4.3$, respectively. The vascular risk factors distribution and the cognitive and functional tests profiles were those expected in a sample of MCI with SVD (Table 1). No patient was on antidementia drugs before or during the trial. Comparisons between attention training and standard care groups showed that there were not statistically significant differences in baseline demographics, vascular risk factors, and global cognitive and functional status (Table 1).

Results for the primary functional outcomes are shown in Table 2. Considering both the $\Delta s$ and the clinically significance approaches, treated and nontreated patients did not differ significantly in any of the functional or quality of life measures after 6 or 12 months.

Results for the cognitive secondary outcomes are shown in Table 3. Using the $\Delta \mathrm{s}$ approach, we found a statistically significant difference between treated and non-treated patients in the Rey Auditory-Verbal Learning Test immediate recall (working memory) when comparing 6 versus 12 months (effect size: Hedges' $g=0.72$ ) and baseline versus 12 months (effect size: Hedges' $g=0.67$ ) in favor of treated patients. Taking into consideration the clinically significance approach, we found that percentages of patients having a stable or better evaluation were significantly higher in treated patients compared to non-treated ones both in the Visual search test (effect size: $\varphi=0.32$ ) and in the Rey-Osterrieth Complex Figure immediate copy (effect size: $\varphi=0.34$ ) in the 6 versus 12 months' comparison.

Out of the 43 enrolled patients, 3/21 (14\%) in the treatment group and 5/22 (23\%) in the standard care group were diagnosed as demented at 1-year follow-up, 17/21 treated (81\%) and 17/22 non-treated $(77 \%)$ remained $\mathrm{MCI}$, and 1 treated patient reverted to normal cognitive function. The chi square test 


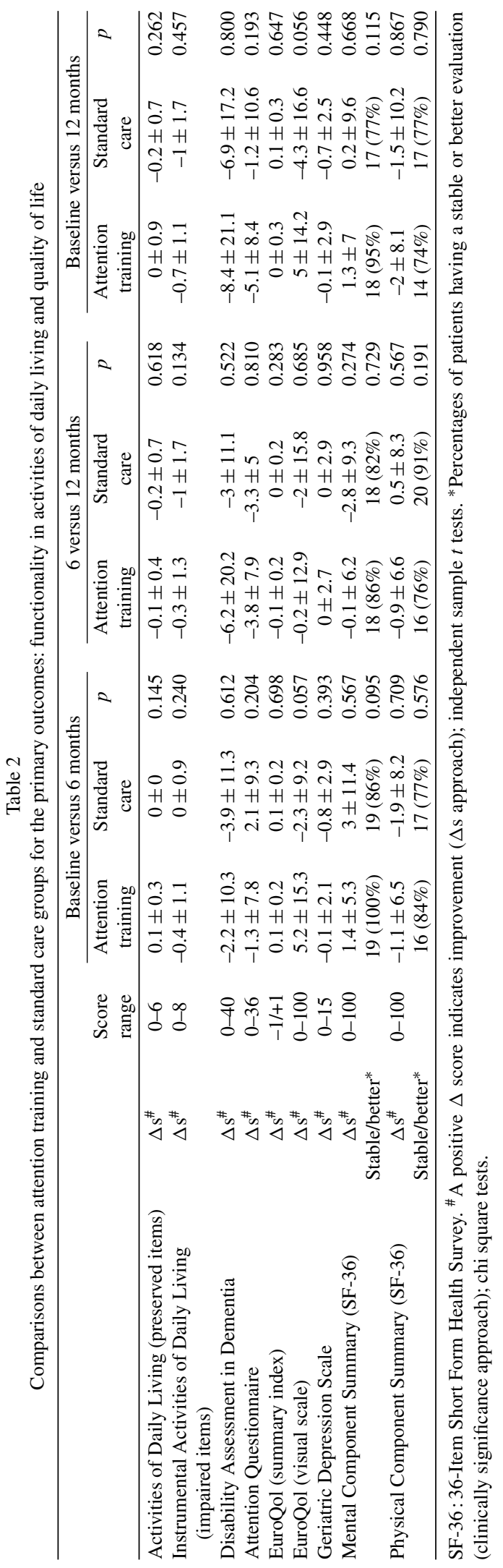

for the association between treatment and dementia diagnosis was not statistically significant.

RsfMRI data were available in 22 patients (12 treated and 10 non-treated) after the exclusion of patients for technical reasons or head movement (in terms of mean voxel absolute displacement) greater than $2 \mathrm{~mm}$, and patients with incidental non-lacunar infarcts in the cerebral cortex, cerebellum, or brainstem to avoid a possible confounding effect on rsfMRI analysis.

In the attention training group, the difference of Z-transformed ReHo data between follow-up and baseline was significantly greater than in the standard care group in 3 clusters whose maximal statistic was in the vermis VIIIb and in the bilateral VIIb lobule of cerebellum, respectively (Supplementary Table 1 and Fig. 2). In each cluster, the mean of the difference of the Z-transformed ReHo (follow-up - baseline) was positive for the attention training group (ReHo increased over time) and negative for the standard care group (ReHo decreased over time) (Fig. 2).

\section{DISCUSSION}

RehAtt is the first-ever randomized clinical trial using cognitive training to improve functional outcome and cognitive performances of patients with MCI and SVD.

We were not able to detect a favorable effect of the APT-II program on functional status and quality of life in our elderly sample. We observed instead some moderately beneficial effects with medium to large effect sizes on a few cognitive tests evaluating attention and working memory domains. Moreover, APT-II program also produced an increased activity in brain circuits involved in cognitive processes.

Overall, the trial has, therefore, to be considered inconclusive. The main possible reason for this could be obviously treatment inefficacy. One could alternatively hypothesize that the trial could be underpowered due to the limited sample size, and therefore effects on quality of life and activities of daily living were hard to detect. Also, treatment duration and intensity may have played a role. It is not known whether the same program implemented for longer periods or a more intensive treatment could have produced different effects. More intensive treatments however generate compliance issues.

Notably, in our study, the non-treated group remained globally stable in functional and cognitive performances. This behavior appears different 


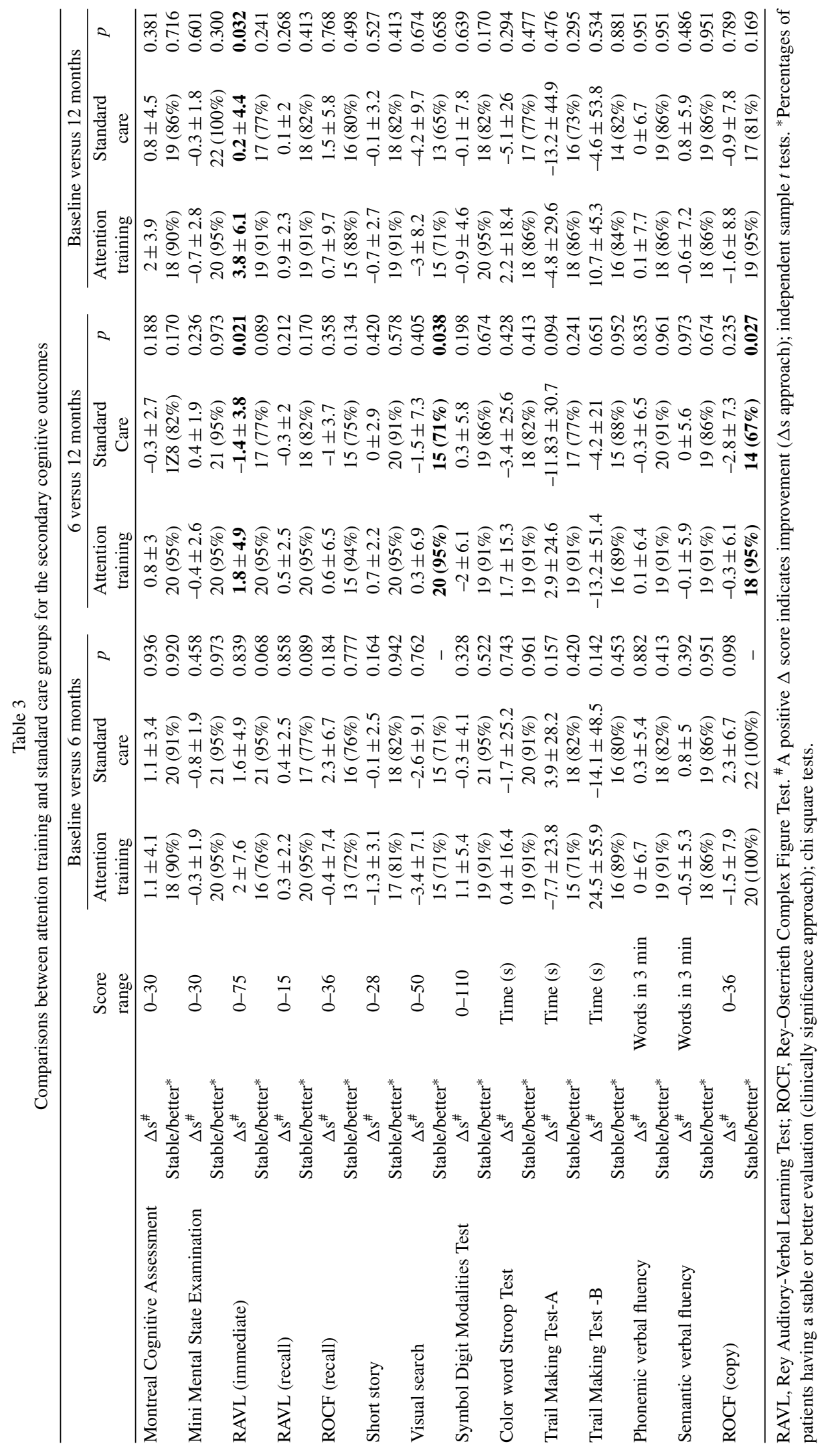




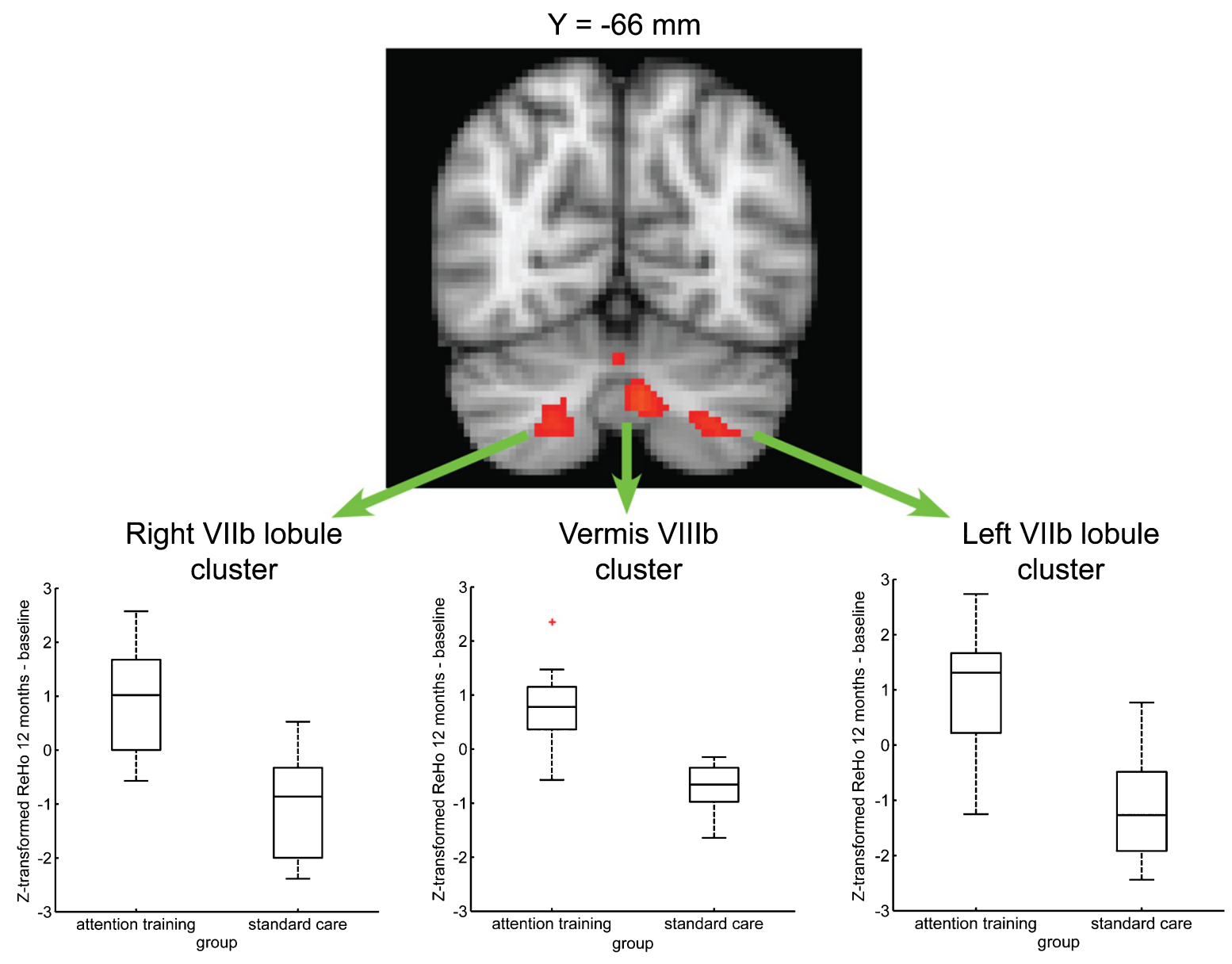

Fig. 2. Improvement in long-term brain activity measured by ReHo of rsfMRI data. Between-group voxel-wise map (TFCE) showing significant $(p<0.05$, corrected) evidence for a greater Z-transformed ReHo difference (12 months - baseline) in cerebellar areas (vermis VIIIb and bilateral VIIb lobule) involved in cognitive processes in the attention training group as compared to standard care group. The map is overlaid on the MNI152 template. In each cluster, the mean of the difference of the Z-transformed ReHo was positive for the attention training group (ReHo increased over time) and negative for the standard care group (ReHo decreased over time).

from the natural history of MCI patients with SVD in whom a decline is usually seen. This behavior of the control group might have attenuated the differences between treated and non-treated patients. Interestingly, this behavior of the placebo groups has been seen also in other trials in similar patients [18].

One last point concerns the mean age of our population, that is quite old, and the choice to include patients with a moderate to severe degree of WMH. Whether the same approach could have different effect either in younger age-groups of patients or in a population with a lesser burden of WMH remains to be explored.

A few considerations related to this type of cognitive treatment can be made. First, the APT-II program is demanding in terms of patient compliance. This notwithstanding, the rate of drop-out was extremely low in our study. However, these cognitive training approaches can be proposed only to well-motivated patients and as reflected by the high selection rate recorded in our study in which a not negligible number of patients refused to take part.

Finally, as typical of cognitive training approaches, the APT-II program places increasing demands on working memory systems and complex attentional control. The lack of efficacy of treatment in our study may also depend on the fact that a direct attention cognitive training could be a challenging approach for elderly MCI patients, thus reducing the possibility to achieve a generalizable effect. In our elderly cohort, it could be hypothesized that our attention training program could have produced 
some potentially beneficial effects only on those tests whose performance is influenced by basic attentional control underlying working memory. Future trials on elderly MCI patients should verify the efficacy of cognitive interventions that are less demanding and more closely related to everyday life tasks, such as cognitive rehabilitation approaches based on meta-cognitive strategies' learning.

Despite the null effect on primary outcomes, the APT-II treatment induced beneficial, although preliminary, effects on some cognitive measures. Moreover, it was associated with a greater difference between follow-up and baseline in the synchronization of activity of some cerebellar areas. This change of cerebellar activity involved the vermis VIIIb and the VIIb lobule, bilaterally. These cerebellar regions are among those known to have projections from and to the prefrontal cortex (cerebello-thalamo-cortical loop) and considered crucial for the contribution of the cerebellum to cognition $[19,20]$. Functional topography of the cerebellum indicates that the left VIIb lobule is activated during executive function tasks [21] while VIIb and VIII lobules supports working memory processes [22]. Since executive functional tasks also activate several areas located throughout the cerebellum, with no clear lateralization or lobular pattern [21], and taking into account that neuroplasticity phenomena associated with SVD can imply variable rearrangement (expansion and dislocation) of functional areas [23], we hypothesize that the above ReHo differences may reflect possible compensatory phenomena induced by the treatment. The increased activity in VIIb and VIII lobules in treated patients appears also in line with the improvement in one working memory task. However, these rsfMRI results need to be taken with caution considering the high drop-out rate on this examination.

The above rsfMRI finding, although preliminary, is in agreement with the involvement of cerebellum in the pathophysiology of cognitive deficits in patients with MCI and SVD revealed by ReHo analysis of rsfMRI data [24].

Of note, these increases in brain activity and improvement in cognitive testing were sustained because they were detected 6 months after the cessation of the cognitive program. Taken together, these data on secondary outcomes seem to outline that the cognitive treatment with the APT-II program has some potential effects whose clinical significance in terms of functionality and quality of life is poorly defined.
Limitations of our study need to be considered. The limited sample size and the lack of a priori power analysis partially attenuated the statistical validity of our preliminary results that will have to be further investigated in future confirmatory trials planned according to effect sizes derived from the present study.

Another possible limitation may be the lack of evaluation of markers of Alzheimer's disease (cerebrospinal fluid biomarkers or positron emission tomography) to better define the etiology of our sample, and rule out the degenerative component. Nevertheless, this patient sample represents what is frequently encountered in clinical practice, and the study was designed to offer therapeutic options that could be easily implemented in a real-world clinical setting without requiring advanced diagnostic techniques not easily available.

In conclusion, the APT-II program could have the potential to produce some beneficial effects on cognitive status and brain activity in patients with MCI and SVD. However, the RehAtt study has been conceived and designed as a pilot study, and our preliminary results primarily represent a methodological background for the development of other clinical trials in larger patient cohorts.

\section{ACKNOWLEDGMENTS}

The RehAtt study was funded by Tuscany region and Italian Ministry of Health under Grant Aimed Research Call 2010 (Bando Ricerca Finalizzata 2010).

Raffaella Valenti, Eleonora Della Rocca, and Emilia Salvadori have been supported by the RehAtt study funds.

Authors' disclosures available online (http://j-alz. com/manuscript-disclosures/17-0428r1).

\section{SUPPLEMENTARY MATERIAL}

The supplementary material is available in the electronic version of this article: http://dx.doi.org/ 10.3233/JAD-170428.

\section{REFERENCES}

[1] Stephan BC, Matthews FE, Khaw KT, Dufouil C, Brayne C (2009) Beyond mild cognitive impairment: Vascular cognitive impairment, no dementia (VCIND). Alzheimers Res Ther 1, 4.

[2] Pantoni L, Gorelick P (2011) Advances in vascular cognitive impairment 2010. Stroke 42, 291-293. 
[3] O'Brien JT, Erkinjuntti T, Reisberg B, Roman G, Sawada T, Pantoni L, Bowler JV, Ballard C, DeCarli C, Gorelick PB, Rockwood K, Burns A, Gauthier S, DeKosky ST (2003) Vascular cognitive impairment. Lancet Neurol 2, 89-98.

[4] Wentzel C, Rockwood K, MacKnight C, Hachinski V, Hogan DB, Feldman H, Østbye T, Wolfson C, Gauthier S, Verreault R, McDowell I (2001) Progression of impairment in patients with vascular cognitive impairment without dementia. Neurology 57, 714-716.

[5] Sohlberg M, Mateer CA (1996) Attention Process TrainingII (APT-II), Association for Neuropsychological Research and Development, Puyallup WA.

[6] Salvadori E, Poggesi A, Valenti R, Della Rocca E, Diciotti S, Mascalchi M, Inzitari D, Pantoni L (2016) The rehabilitation of attention in patients with mild cognitive impairment and brain subcortical vascular changes using the Attention Process Training-II. The RehAtt Study: Rationale, design and methodology. Neurol Sci 37, 1653-1662.

[7] Winblad B, Palmer K, Kivipelto M, Jelic V, Fratiglioni L, Wahlund LO, Nordberg A, Bäckman L, Albert M, Almkvist O, Arai H, Basun H, Blennow K, de Leon M, DeCarli C, Erkinjuntti T, Giacobini E, Graff C, Hardy J, Jack C, Jorm A, Ritchie K, van Duijn C, Visser P, Petersen RC (2004) Mild cognitive impairment - beyond controversies, towards a consensus: Report of the International Working Group on Mild Cognitive Impairment. J Intern Med 256, 240-246.

[8] Salvadori E, Poggesi A, Valenti R, Pracucci G, Pescini F, Pasi M, Nannucci S, Marini S, Del Bene A, Ciolli L, Ginestroni A, Diciotti S, Orlandi G, Di Donato I, De Stefano N, Cosottini M, Chiti A, Federico A, Dotti MT, Bonuccelli U, Inzitari D, Pantoni L, VMCI-Tuscany Study Group (2016) Operationalizing mild cognitive impairment criteria in small vessel disease: The VMCI-Tuscany Study. Alzheimers Dement 12, 407-418.

[9] Pantoni L, Basile AM, Pracucci G, Asplund K, Bogousslavsky J, Chabriat H, Erkinjuntti T, Fazekas F, Ferro JM, Hennerici M, O’Brien J, Scheltens P, Visser MC, Wahlund LO, Waldemar G, Wallin A, Inzitari D (2005) Impact of age-related cerebral white matter changes on the transition to disability - the LADIS study: Rationale, design and methodology. Neuroepidemiology 24, 51-62.

[10] Salvadori E, Poggesi A, Pracucci G, Inzitari D, Pantoni L, VMCI-Tuscany Study Group (2015) Development and psychometric properties of a neuropsychological battery for mild cognitive impairment with small vessel disease: The VMCI-Tuscany Study. J Alzheimers Dis 43, 1313-1323.
[11] Katz S, Ford AB, Moskowitz RW, Jackson BA, Jaffe MW (1963) Studies of illness in the aged. The index of ADL: A standardized measure of biological and psychosocial function. JAMA 185, 914-919.

[12] Lawton MP, Brody EM (1969) Assessment of older people: Self-maintaining and instrumental activities of daily living. Gerontologist 9, 179-186.

[13] Gélinas I, Gauthier L, McIntyre M, Gauthier S (1999) Development of a functional measure for persons with Alzheimer's disease: The Disability Assessment for Dementia. Am J Occup Ther 53, 471-481.

[14] Ware JE, Sherbourne CD (1992) The MOS 36-item shortform health survey (SF-36). I. Conceptual framework and item selection. Med Care 30, 473-483.

[15] Rabin R, de Charro F (2001) EQ-5D: A measure of health status from the EuroQol Group. Ann Med 33, 337-343.

[16] Yesavage JA (1988) Geriatric Depression Scale. Psychopharmacol Bull 24, 709-711.

[17] American Psychiatric Association (2013) Diagnostic and Statistical Manual of Mental Disorders 5th Ed, American Psychiatric Publishing, Arlington VA.

[18] Wilkinson D, Doody R, Helme R, Taubman K, Mintzer J, Kertesz A, Pratt RD, Donepezil 308 Study Group (2003) Donepezil in vascular dementia: A randomized, placebocontrolled study. Neurology 61, 479-486.

[19] Buckner RL (2013) The cerebellum and cognitive function: 25 years of insight from anatomy and neuroimaging. Neuron 80, 807-815.

[20] Stoodley CJ (2012) The cerebellum and cognition: Evidence from functional imaging studies. Cerebellum 11, 352-365.

[21] Stoodley CJ, Schmahmann JD (2009) Functional topography in the human cerebellum: A meta-analysis of neuroimaging studies. Neuroimage 44, 489-501.

[22] Marvel CL, Desmond JE (2010) Functional topography of the cerebellum in verbal working memory. Neuropsychol Rev 20, 271-279.

[23] Sun YW, Qin LD, Zhou Y, Xu Q, Qian LJ, Tao J, Xu JR (2011) Abnormal functional connectivity in patients with vascular cognitive impairment, no dementia: A resting-state functional magnetic resonance imaging study. Behav Brain Res 223, 388-394.

[24] Diciotti S, Orsolini S, Salvadori E, Giorgio A, Toschi N, Ciulli S, Ginestroni A, Poggesi A, De Stefano N, Pantoni L, Inzitari D, Mascalchi M, VMCI Tuscany Investigators (2017) Resting state fMRI regional homogeneity correlates with cognition measures in subcortical vascular cognitive impairment. J Neurol Sci 373, 1-6. 